\title{
Variabilidade da freqüiência cardíaca em cães anestesiados com infusão contínua de propofol e sufentanil
}

\author{
[Heart rate variability in dogs anesthetized with propofol and sufentanil] \\ R. Carareto $^{1}$, M.G. Sousa ${ }^{1}$, J.C. Zacheu ${ }^{2}$, A.J.A. Aguiar ${ }^{3}$, A.A. Camacho ${ }^{4}$ \\ ${ }^{1}$ Aluno de pós-graduação - FCAV-UNESP - Jaboticabal, SP \\ ${ }^{2}$ Faculdade de Jaguariúna - FAJ - Jaguariúna, SP \\ ${ }^{3}$ Curso de Medicina Veterinária - UNESP - Araçatuba, SP - \\ ${ }^{4}$ Faculdade de Ciências Agrárias e Veterinárias - UNESP \\ Via de Acesso Prof. Paulo Donato Castellane, s/n \\ 14884-900 - Jaboticabal, SP
}

\begin{abstract}
RESUMO
Foram avaliados os efeitos do propofol associado ao sufentanil sobre o balanço das atividades simpática e parassimpática do coração, investigando-se um possível efeito dose dependente do opióide. Analisou-se a variabilidade da freqüência cardíaca (VFC) de 12 cães adultos pré-medicados com maleato de acepromazina e anestesiados com propofol e três doses diferentes de sufentanil, que variou de 0,025 a $0,1 \mu \mathrm{g} / \mathrm{kg} / \mathrm{min}$. Registrou-se o eletrocardiograma 15 minutos após a medicação pré-anestésica e 15, 30, 60, 90 e 120 minutos após a indução anestésica. A VFC foi calculada no domínio da freqüência, mediante análise de 10 intervalos RR consecutivos. Houve redução acentuada da freqüência cardíaca, mas a VFC permaneceu relativamente inalterada.
\end{abstract}

Palavras-chave: cão, anestesia intravenosa, opióides, tônus parassimpático, freqüência cardíaca

\begin{abstract}
The effects of propofol and sufentanil on cardiac sympathetic and parasympathetic balance were studied, in order to evaluate if sufentanil plays a role in this balance. The heart rate variability of 12 adult dogs was assessed, after premedication with acepromazine and anesthetized with propofol and three different doses of sufentanil, ranging from 0.025 to $0.1 \mu \mathrm{g} / \mathrm{kg} / \mathrm{min}$. Electrocardiograms were recorded 15 minutes after premedication and 15, 30, 60, 90, and 120 minutes after anesthetic induction. Heart rate variability was calculated in frequency domain through the analysis of 10 consecutive RR intervals. Results showed an absence of important changes in heart rate variability, although a significant decrease in heart rate was observed.
\end{abstract}

Keywords: dog, intravenous anesthesia, opioids, parasympathetic tone, heart rate

\section{INTRODUÇÃO}

A análise da variabilidade da freqüência cardíaca (VFC) é um método simples e não-invasivo que avalia a atividade do sistema nervoso autônomo sobre o coração, permitindo determinar alterações simpatovagais (Guzzetti et al., 2001).
Assim, um aumento do tônus do sistema nervoso autônomo simpático é acompanhado por redução dos valores da VFC, enquanto que um incremento do tônus do sistema nervoso autônomo parassimpático resulta em aumento dos valores da VFC (Montano et al., 2001).

$\mathrm{O}$ propofol é um agente hipnótico amplamente utilizado em anestesia intravenosa. Mesmo após

Recebido em 10 de fevereiro de 2005

Aceito em 27 de dezembro de 2006

E-mail: robertacarareto@hotmail.com 
sucessivas aplicações, o propofol apresenta mínimos efeitos cumulativos, favorecendo uma recuperação rápida e tranqüila (Hall e Clark, 2000). Apesar da baixa ocorrência de efeitos colaterais, sabe-se que a anestesia realizada com o propofol é freqüentemente associada com decréscimo da pressão arterial e redução da freqüência cardíaca (Smith et al., 1994). Entretanto, o real efeito do propofol sobre tônus cardíaco simpático e parassimpático ainda é questionável (Noriaki et al., 2003). Alguns pesquisadores afirmam que a anestesia com propofol reduz menos o tônus parassimpático que o simpático (Deutschman et al., 1994), enquanto outros concluíram que há um predomínio do sistema nervoso simpático sobre o parassimpático (Scheffer et al., 1993).

Com relação ao sufentanil sabe-se que este opióide possui discreta atividade simpatolítica e vagotônica, provocando redução dose-dependente da freqüência cardíaca e aumento da duração do potencial de ação nas fibras de Purkinje (Blair et al., 1989; Bovill, 1993). No caso do fentanil, a análise da VFC mostrou que ocorre depressão do sistema nervoso simpático e manutenção da atividade do parassimpático, sendo tal modulação independente da dose infundida ou da velocidade de administração (Zickmann et al., 1996).

São exíguas as informações sobre o efeito da anestesia intravenosa total realizada com a associação de propofol e sufentanil sobre o sistema nervoso autônomo canino. Com base nisso, o estudo foi desenvolvido para avaliar os efeitos dessa associação de fármacos sobre o balanço entre as atividades simpática e parassimpática do coração, investigando-se um possível efeito dose dependente do sufentanil.

\section{MATERIAL E MÉTODOS}

Utilizaram-se 12 cadelas adultas, sem raça definida, clinicamente sadias e com peso de $18,21 \pm 2,81 \mathrm{~kg}$ ( $\overline{\mathbf{x}} \pm \mathrm{DP}$ ), evitando-se animais gestantes ou em fase de estro. Cada animal foi anestesiado três vezes, com intervalo de uma semana entre cada procedimento, sendo utilizada uma dose diferente de sufentanil em cada procedimento anestésico.

Todos os cães receberam maleato de acepromazina ${ }^{1}$ como medicação pré-anestésica, na dose de $0,05 \mathrm{mg} / \mathrm{kg}$, aplicada pela via intravenosa. Em seguida, um cateter ${ }^{2}$ foi implantado na veia cefálica

\footnotetext{
${ }^{1}$ Acepran 0,2\% - Univet - São Paulo - SP

${ }^{2}$ Angiocath 20G - Becton Dickinson - Juiz de Fora - MG
}

esquerda, sendo acoplado a uma válvula de três vias e duas bombas de infusão intravenosa ${ }^{3}$, uma delas para a administração de $\operatorname{propofol}^{4} \mathrm{e}$ a outra para o citrato de sufentanil ${ }^{5}$. A indução anestésica foi realizada com a administração de propofol, na dose de $5 \mathrm{mg} / \mathrm{kg}$, lentamente, pela via intravenosa. A manutenção anestésica se iniciou após a indução, com a administração de propofol $(0,2 \mathrm{mg} / \mathrm{kg} /$ minuto IV) associado ao citrato de sufentanil, em três diferentes doses de acordo com os grupos: (G1) $0,025 \mu \mathrm{g} / \mathrm{kg} / \mathrm{minuto}$, (G2) $0,05 \mu \mathrm{g} / \mathrm{kg} /$ minuto e (G3) $0,1 \mu \mathrm{g} / \mathrm{kg} /$ minuto. A infusão de sufentanil, assim como do propofol, teve a duração de 120 minutos, sendo ambas interrompidas simultaneamente.

Instituiu-se ventilação controlada, de modo a manter os valores de $\mathrm{ETCO}_{2}$ entre 35 e $45 \mathrm{mmHg}$. Para tanto, utilizou-se um ventilador artificial $^{6}$ ciclado a volume, ajustado na freqüência respiratória de 10 movimentos respiratórios por minuto, volume corrente de $20 \mathrm{ml} / \mathrm{kg}$ e relação inspiração/expiração de $1: 2$, sendo o tempo inspiratório de dois segundos, mantendo-se a ventilação a pressão positiva intermitente, com oxigênio a $100 \%$.

Em situações em que freqüência cardíaca (FC) tornou-se inferior a 60 batimentos por minuto e a pressão arterial média obtida de forma invasiva inferior a $60 \mathrm{mmHg}$, administrou-se sulfato de atropina $^{7}$, na dose de $0,022 \mathrm{mg} / \mathrm{kg}$, pela via intramuscular.

Em todos os grupos, efetuou-se registro do eletrocardiograma em derivação DII com emprego de eletrocardiógrafo monocanal ${ }^{8}$, calibrado na velocidade de $25 \mathrm{~mm} / \mathrm{s}$ e com sensibilidade ajustada para $1 \mathrm{~cm}$ igual a $1 \mathrm{mV}$, sendo cada um dos quatro eletrodos posicionados individualmente em cada membro do animal. Procedeu-se o registro eletrocardiográfico 15 minutos após a medicação pré-anestésica (M0) e 15 (M1), 30 (M2), 60 (M3), 90 (M4) e 120 (M5) minutos após a indução anestésica, sendo registrado o traçado por pelo menos um minuto em cada um dos momentos.

A VFC foi calculada no domínio da freqüência, por meio da análise de 10 intervalos RR consecutivos

\footnotetext{
${ }^{3}$ Samtronic 680 e Samtronic 550T2-Samtronic - São Paulo SP

${ }^{4}$ Diprivan 1\% - AstraZeneca - Cotia - SP

${ }^{5}$ Sufenta $75 \mu \mathrm{g} / \mathrm{ml}$ - Janssen-Cilag - São José dos Campos SP

${ }^{6}$ Ventilador mecânico Volumétrico/Pressométrico 676 - K. Takaoka - São Paulo - SP

${ }^{7}$ Atropion $0,25 \mathrm{mg} / \mathrm{ml}$ - Laboratórios Teuto - Anápolis - GO

${ }^{8}$ Eletrocardiógrafo CD-188 - Berger - São Paulo - SP
} 
(em milissegundos), a partir dos quais calculou-se a variância amostral, cujo logaritmo natural (neperiano) representa a VFC propriamente dita (Tárraga, 2002). Para tanto, utilizou-se a seguinte equação matemática:

$V F C=\log _{e}\left(\frac{n \sum_{i=1}^{n} x_{i}^{2}-\left(\sum_{i=1}^{n} x_{i}\right)}{n(n-1)}\right)$, em que:

$\mathrm{VFC}=$ variabilidade da freqüência cardíaca;

$\log _{\mathrm{e}}=\log _{\text {aritmo natural (neperiano); }}$

$\mathrm{n}=$ número de intervalos $\mathrm{RR}$ analisados;

$\mathrm{x}=$ intervalo $\mathrm{RR}$ (em milisegundos)
O delineamento experimental foi em parcelas subdivididas. Os dados foram submetidos à análise de variância, seguida do pós-teste Tukey-Kramer para determinar em quais momentos tais variações ocorriam. Para a comparação entre os grupos, procedeu-se o teste Student-Newman-Keuls.

\section{RESULTADOS E DISCUSSÃO}

Os resultados da VFC e intervalo RR estão expressos na Tab. 1.

Tabela 1. Variabilidade da freqüência cardíaca (VFC) e intervalo RR médio (RRm) em cães anestesiados com infusão contínua de propofol e doses crescentes de sufentanil

\begin{tabular}{|c|c|c|c|c|c|c|c|c|}
\hline & Grupo & $\begin{array}{c}\text { M0 } \\
\text { Basal }\end{array}$ & $\begin{array}{c}\mathrm{M} 1 \\
15 \mathrm{~min}\end{array}$ & $\begin{array}{c}\mathrm{M} 2 \\
30 \mathrm{~min}\end{array}$ & $\begin{array}{c}\text { M3 } \\
60 \mathrm{~min}\end{array}$ & $\begin{array}{c}\text { M4 } \\
90 \mathrm{~min}\end{array}$ & $\begin{array}{c}\text { M5 } \\
120 \mathrm{~min}\end{array}$ & $\mathrm{P}$ \\
\hline \multirow{3}{*}{ VFC } & G1 & $9,35 \pm 1,84$ & $8,03 \pm 2,07$ & $8,71 \pm 1,55$ & $8,56 \pm 1,68 \mathrm{ab}$ & $8,34 \pm 1,89 a$ & $9,36 \pm 1,40 \mathrm{a}$ & 0,3507 \\
\hline & G2 & $10,67 \pm 1,90$ & $9,23 \pm 1,65$ & $9,77 \pm 1,18$ & $9,89 \pm 1,89 \mathrm{a}$ & $8,86 \pm 2,28 \mathrm{a}$ & $8,78 \pm 2,23 \mathrm{ab}$ & 0,1316 \\
\hline & G3 & $9,51 \pm 2,20$ & $8,64 \pm 1,18$ & $8,36 \pm 1,76$ & $7,08 \pm 2,22 * b$ & $6,67 \pm 1,43 * b$ & $7,01 \pm 2,72 * b$ & 0,0041 \\
\hline \multirow{3}{*}{$\begin{array}{l}\mathrm{RRm} \\
\text { (milisegundos) }\end{array}$} & G1 & $795,1 \pm 237,3$ & $761,4 \pm 213,2 \mathrm{a}$ & $906,4 \pm 189,6 \mathrm{a}$ & $1056,0 \pm 193,9 *$ & $1042,2 \pm 156,7 * a$ & $1045,0 \pm 198,2 *$ & 0,0003 \\
\hline & $\mathrm{G} 2$ & $882,2 \pm 206,3$ & $1027,9 \pm 224,4 \mathrm{~b}$ & $1143,6 \pm 156,8^{*} \mathrm{~b}$ & $1099,5 \pm 196,3 *$ & $997,5 \pm 154,8 \mathrm{ab}$ & $1041,0 \pm 163,1$ & 0,0221 \\
\hline & G3 & $864,8 \pm 199,7$ & $1072,2 \pm 253,4 \mathrm{~b}$ & $1113,7 \pm 275,0 \mathrm{~b}$ & $976,0 \pm 225,5$ & $826,0 \pm 297,7 b$ & $812,0 \pm 318,7$ & 0,0209 \\
\hline
\end{tabular}

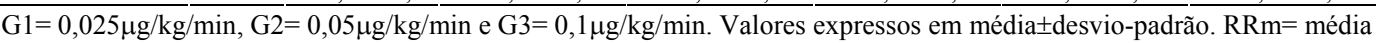
aritmética de 10 intervalos RR consecutivos. *Diferem de $\mathrm{MO}(\mathrm{P}<0,05)$. Valores seguidos por letras distintas na mesma coluna diferem entre si $(\mathrm{P}<0,05)$.

O intervalo RR foi significativamente menor no G1 em relação ao G2 e G3 em M1 e M2. Em M4, o G3 foi significativamente menor que o G1. Na comparação entre grupos, observa-se que no G1 as médias foram significativamente maiores a partir de $\mathrm{M} 3$, enquanto que para o $\mathrm{G} 2$, as médias de M2 e M3 foram significativamente maiores que as demais, e no G3 houve gradativa redução a partir de M3, o que pode estar relacionado à aplicação do sulfato de atropina por via intramuscular em 50\% dos animais por volta dos 30 minutos de anestesia. Esse procedimento foi necessário devido à redução acentuada da FC assim como da pressão arterial média em alguns momentos no G3. Hughes e Nolan (1999) e Freye et al. (2000) também observaram bradicardia durante anestesia com propofol e sufentanil em cães e atribuíram essa ocorrência ao aumento da atividade vagal, o que pode justificar o acréscimo significativo do intervalo RR no G1 a partir de M3.

Em relação à VFC, observaram-se diferenças significativas entre G3 e os demais grupos a partir de M3 (Tab. 1).

Em M3, os valores da VFC foram significativamente menores no G3 quando comparado com o G2, enquanto que em M4, G3 diferiu dos demais grupos. Em M5, o G3 apresentou valores significativamente menores em relação ao G1. É provável que a menor VFC nos momentos finais no G3 relacione-se ao efeito parassimpatolítico do sulfato de atropina (Kawamoto et al., 1996) administrado em 50\% dos animais por volta dos 30 minutos de anestesia. Noriaki et al. (2003) relataram que o propofol reduz o tônus parassimpático em menor escala que o simpático, resultando em predominância do sistema nervoso parassimpático, o que leva a alterações hemodinâmicas e decréscimo da FC.

Neste estudo observou-se redução da FC em todos os grupos, sendo claramente observada um efeito dose dependente do propofol. Entretanto, a aplicação do sulfato de atropina contribuiu para que a redução da FC não fosse significativa em G3, sendo inclusive, observado incremento a partir de M3, provavelmente refletindo a preponderância do sistema nervoso simpático decorrente da ação da atropina sobre o parassimpático. Dessa forma, notou-se maior estabilidade da FC e, conseqüentemente, menor VFC, de forma similar aos estudos realizados por Scheffer et al. (1993) e Kawamoto et al. (1996).

Em G3, houve redução significativa da VFC em relação ao valor basal a partir de M3. A ausência de alterações significativas na VFC em G1 e G2 é um 
achado importante, pois se sabe que fármacos como fentanil e sufentanil deprimem a atividade do sistema nervoso autônomo simpático, mas não a atividade do parassimpático, sendo esperado incremento na VFC durante a anestesia com esses opióides (Zickamann et al., 1996). Especificamente para o G3, o acréscimo da $\mathrm{FC}$ e, portanto, redução da VFC, eventualmente não seria observada na ausência do sulfato de atropina. Há que se considerar que a utilização do sulfato desse anticolinérgico pode ter mascarado os efeitos da maior dose de sufentanil sobre a atividade do sistema nervoso autônomo. Em G1 e G2, a VFC foi semelhante, não existindo diferenças significativas entre os grupos na maioria dos momentos. A ausência de diferenças entre grupos pode ser atribuída ao um possível efeito dose dependente do propofol na estimulação do tônus parassimpático (Noriak et al., 2003) e do sufentanil no aumento da atividade vagal (Freye et al., 2000).

\section{CONCLUSÕES}

Evidenciou-se ausência de variações importantes na VFC, apesar da acentuada redução na FC. Aparentemente o uso de sufentanil em doses de até $0,05 \mu \mathrm{g} / \mathrm{kg} /$ minuto não exerce efeito sobre o tônus parassimpático de cães anestesiados pelo propofol.

\section{REFERÊNCIAS BIBLIOGRÁFICAS}

BLAIR, J.R.; INTRONA, R.P.; ADAMS, R.J. et al. Cardiac electrophysiology effects of fentanyl and sufentanil in canine cardiac purkinje. Anesthesiology, v.4, p.565-570, 1989.

BOVILL, J.G. Os opióides na anestesia intravenosa. In: DUNDEE, J.W.; WYANT, G.M. Anestesia intravenosa. 2.ed. Rio de Janeiro: Revinter, 1993. p.192-229.

DEUTSCHMAN, C.S.; HARRIS, A.P.; FLEIAHER, L.A. Changes in heart variability under propofol anesthesia: A possible explanation for propofol-induced bradicardia. Anesth. Analg., v.79, p.373-377, 1994.

FREYE, E.; SCHMIDHAMMER, H.; LATASCH, L. 14-methoxymetopon, a potent opioid agonist, induces no respiratory depression, less sedation, and less bradicardia than sufentanil in the dog. Anesth. Analg., v.90, p.1359-1364, 2000.
GUZZETTI, S.; MAGATELLI, R.; BORRONI, E. et al. Heart rate variability in chronic heart failure. Auton. Neuroscie., v.90, p.102-105, 2001.

HALL, L.W.; CLARKE, K.W. General pharmacology of intravenous anaesthetics agents. In: VETERINARY ANAESTHESIA. 10.ed. London England: Baillère Tindall, 2000. p.422.

HUGHES, J.M.L.; NOLAN, A.M. Total intravenous anesthesia in greyhounds: pharmacokinetics of propofol and fentanyl - a preliminary study. Vet. Surg., v.6, p.513-524, 1999.

KAWAMOTO, M.; MATSUMOTO, C.; YUGE, O. Atropine premedication attenuates heart rate variability during high thoracic epidural anesthesia. Acta Anaesthesiol. Scand., v.40, p.1132-1137, 1996.

MONTANO, N; COGLIATI, C.; SILVA, V.J.D. et al. Sympathetic rhythms and cardiovascular oscillations. Auton. Neuroscie., v.90, p.29-34, 2001.

NORIAK, K.; NAOTUKI, H.; SAORI, K. et al. Differential effects of propofol and sevoflurane on heart rate variability. Anesthesiology, v.98, p.34-40, 2003.

SCHEFFER, G.J.; TEN-VOORDE, B.J., KAREMAKER, J.M. et al. Effects of thiopentone, etomidate and propofol on beat-to-beat cardiovascular signals in man. Anaesthesia, v.48, p.849-855, 1993.

SMITH, I.; WHITE, P.F.; NATHANSON, M. et al. Propofol: An update on its clinical use. Anesthesiology, v.81, p.1005-1043, 1994.

TÁRRAGA, K.M. Avaliação clínica da utilização de bloqueadores farmacológicos no estudo dos parâmetros cardiorrespiratórios e da variabilidade da freqüencia cardiaca em modelos experimentais de miocardiopatia em cães. 2002. 87f. Tese (Doutorado em Patologia Experimental e Comparada) - Faculdade de Medicina Veterinária e Zootecnia, Universidade de São Paulo, São Paulo.

ZICKMANN, B.; HOFMANN, H.C.; POTTKAMPER, $\mathrm{C}$. et al. Changes in heart rate variability during induction of anesthesia with fentanyl and midazolam. J. Cardiothorac. Vasc. Anesth., v.10, p.609-613, 1996. 